\title{
A TUNABLE X-RAY MICROPROBE USING SYNCHROTRON RADIATION
}

\author{
Y. Wu, A. C. Thompson, J. H. Underwood, R.D. Giauque, and K. Chapman \\ Lawrence Berkeley Laboratory, Berkeley, CA 94720 \\ M. L. Rivers \\ University of Chicago, Chicago, IL 60637 \\ K. W. Jones \\ Brookhaven National Laboratory, Upton, NY 11973
}

We describe an $x$-ray microprobe using multilayer mirrors. Previously, we had demonstrated a Kirkpatrick-Baez type focusing system working at both 8 and $10 \mathrm{keV}$ and successfully applied it to a variety of applications, including the determination of elemental contents in fluid inclusions. In this paper, we show that the usable excitation energy for this microprobe is not restricted to between 8 and $10 \mathrm{keV}$, and furthermore, it can be simply tuned in operation.

A $10-\mathrm{keV}$ x-ray fluorescence microprobe can be used to measure the concentration of the elements from potassium ( $\mathrm{Z}=19)$ to zinc $(\mathrm{Z}=30$ ) using $\mathrm{K}$-ray lines, and from cadmium $(Z=48)$ to erbium $(Z=68)$ using $L x$-ray lines. There are a number of geologically important elements in the gap between gallium $(\mathrm{Z}=31)$ and silver $(\mathrm{Z}=47)$ and also with $\mathrm{Z}>68$. In order to cover this range, a higher excitation energy is required. On the other hand, for samples that contain major elements with absorption edges lower than the excitation energy , it would be hard to detect other minor elements because of the strong signal from the major elements and the background they produce. In this case, a tunable $\mathrm{X}$-ray source can be used to avoid the excitation of the major elements.

We demonstrate that, with the existing setup, it is possible to tune the excitation energy from $6 \mathrm{keV}$ to $14 \mathrm{keV}$, in this range, the intensity does not decrease by more than one order of magnitude. As an illustration, a geological sample was examined by using two different excitation energies to show the advantage of a tunable source. Finally, we discuss the possibility of further extension of the excitation energy range as well as the possibility of improving the intensity.

*This work was supported by the Director, Office of Energy Research, Office of Basic Energy Sciences, Material Sciences Division, of the U.S. Department of Energy under Contract No. DE-AC03-76SF00098.

\section{DISCLAIMER}

\begin{abstract}
This report was prepared as an ascount of work sponsored by an agency of the United States Government. Neither the United States Government nor any agency bereof, nor any of their employees, makes any warranty. express or implied, or assumes any lezal liability or responsibility for the accurac;, compteteness, or usefulmess of any information, apparatus, product, or process disciosed, or represents that its use would not infringe privately owned rights. Reference berein to any specific commercial product, process, or service by trade name, trademark, manufacturer, or otberwise does not necessarily constitute or imply its endorsement, recommendation, or favoring by the United States Gowetnment or any agency thereof. The views and opinions of authos expressed herein do not necessarily state or refloct those of the United States Government or any agency thereof.
\end{abstract}




\section{Introduction}

Elemental analysis using $\mathrm{X}$-rays from a synchrotron source has many advantages when compared with other techniques (i.e. electron probe, proton probe and ion probe) [1]. Some of the advantages are improved sensitivity and signal to background ratio, ease of operation, and the ability to examine samples in special environments. There have been X-ray microprobe experiments at many synchrotron facilities in the past decade[1-4]. Three basic considerations affect the capability of a microprobe: (1) the energy distribution of the excitation radiation, (2) the intensity or flux level as measured by photons/s and/or photons/s/ $\mathrm{mm}^{2}$ and (3) the spatial resolution. The energy distribution of $\mathrm{X}$-rays from a bending magnet or wiggler magnet synchrotron radiation source is a continuous spectrum which, depending on the machine parameters, can have useful flux up to over $100 \mathrm{keV}$. Using mirrors, filters and crystal monochromators, various energy distributions can be produced from this source. As noted by Sparks[1], the reduced background in the energy region of interest in a spectrum obtained by employing a monochromatic excitation radiation increases the mininum detectable limits (MDL) by up to a factor of $10^{3}$ from that attained using a white excitation source, provided a comparable number of photons in the incident beam can be obtained. In practice, monochromatic radiation is usually obtained by using a silicon double crystal monochromator which produces a beam with a very narrow energy bandpass ( $\mathrm{dE} / \mathrm{E}=10^{-4}$ ) but, unfortunately, a correspondingly much lower intensity. It is clear that a wide-bandpass monochromator $(\mathrm{dE} / \mathrm{E}=0.1$ to 0.01 ) would be very useful. Sometimes it is also desirable to tune the energy of the excitation radiation $[1,2]$. Many focusing geometries have been proposed in the design of $\mathrm{X}$-ray microprobes involving the use of large mirrors or crystals. These components are difficult to fabricate, and therefore fer have been built. More importantly, most of the designs do not have enough demagnification to give a beam spot less than $50 \mu \mathrm{m}$ in diameter withou: the use of a pinhole. Jones and Gordon[4] have recently summarized progress in the development of $\mathrm{X}$-ray microprobes.

In previous experiments, we have demonstrated a simple focusing microprobe system utilizing multilayer mirrors working at $8 \mathrm{keV}[5]$ and $10 \mathrm{keV}[6]$. This system has a relatively wide bandpass and high spatial resolution, which extends the usefulness of $\mathrm{X}$-ray micro- 
probes. For instance, it was used to examine the elemental content in fluid inclusions. Those fluid inclusions, with a size of $10 \sim 100 \mu \mathrm{m}$ in diameter and few $\mu \mathrm{m}$ thick, are bubbles containing aqueous solutions of various minerals buried in a solid substrate like quartz. Study of the composition and distribution of natural fluid inclusions yields important information about the geochemical evolution of the locality. Due to the small size of the bubbles, they can not be easily separated physically from the substrate and it is extremely difficult, or impossible, to measure the elemental composition of the dissolved material. This kind of sample provides an excellent example of the unique capabilities of an X-ray microprobe. However, many geological samples contain elements that have absorption edges higher than $10 \mathrm{keV}$, and in other cases, contain lower- $\mathrm{Z}$ minor elements of interest in a higher- $\mathrm{Z}$ matrix. For these samples a microprobe with selectable excitation energy is desirable so that the elemental sensitivity can be optimized for the particular sample. In this experiment we demonstrated that the same simple microprobe system can be tuned easily across a broad energy range to produce significant improvements in detectability for some samples.

In 1980 Sparks[I] noted that an X-ray microprobe can also be used for diffraction analysis as well as elemental analysis. Later, Ice [7] used a simple collimation method to obtain microbeams for diffraction experiments with relatively low spatial resolution. The high spatial resolution and intensity provided by our focusing system should make a useful diffraction microprobe. Besides diffaction from samples under ambient conditions, a separate class of experiments has emerged with the development of new high pressure techniques. The properties of materials under high pressure is of theoretical interest and, for some materials, great geological interest. Recently, very high static pressures have been achieved using a diamond anvil cell[8]. In this device, a sample is placed between two opposing diamonds with tiny tips facing each other, the diamonds are then pressed together to generate enormous pressures at the sample, which is constrained by a special gasket. The sample size, however, has to be very small - typically less than $1000 \mu \mathrm{m}^{3}$. To study these samples with $\mathrm{X}$-ray diffraction the beam spot size must therefore also be very small so that it measures a volume of the sample that is at constant pressure. In addition, in order to examine the crystal structure of the sample under high pressure in situ, only high energy $\mathrm{X}$-rays (greater than $10 \mathrm{keV}$ ) are useful because the radiation must penetrate the diamonds. In this experiment we also studied the 
feasibility of using a multilayer focusing system for high energy (up to $20 \mathrm{keV}$ ) $\mathrm{X}$-rays.

\section{Experimental}

The experimental arrangement was similar to that used in previous experiments $[5,9]$. Fig. 1 is a schematic of the setup in the X-ray fluorescence microprobe experiment. The multilayer coatings for the mirrors were designed to work at $10 \mathrm{keV}$ and had $d$ spacings of 25.4 and $36.2 \AA$, respectively. At $10 \mathrm{keV}$ the working distance of microprobe is $4.4 \mathrm{~cm}$ from the end of the second focusing mirror and the energy bandpass is less than $1 \mathrm{keV}$.

The alignment procedure for the focusing system consists of first adjusting the glancing angles of both mirrors separately to meet the Bragg condition for reflection at $10 \mathrm{keV}$, then moving each of the mirrors along the beam direction to obtain the minimum focused beam spot size at the sample. The beam size is measured by scanning a sharp knife edge across the beam and measuring the transmitted intensity. The derivative of the transmitted intensity with respect to position gives an upper limit to the actual beam size. After caurying out some experiments using $10 \mathrm{keV}$ radiation, we investigated the performance of this setup at different energies. The process of going to other energies is achieved by using a piece of polypropylene foil free from contaminants as a sample, and measuring the intensity and energy of elastic and Compton-scattered X-rays as the angle and position of the focusing mirrors are changed. The angles of the mirrors are changed alternately in small steps until the new energy setting is reached. A ruby crystal is then inserted at the sample position and the focused spot is viewed with an optical microecope as the two mirror positions along the beam direction are varied. At different energies the reflection angles and therefore the positions of the focused spot in the focusing plane are different. Since the optical system registers the focused spot position, we can then move the sample in order to examine the same spot at different energies.

In this experiment, the best focusing cendition at each energy was not attempted because of the limited beam time. Instead a National Bureau of Standards (NBS) thin glass film Standard Reference Material, SRM 1833, was used, and just the fluorescent spectra at 
different energies were measured. The focused beam intensity can then be found by measuring the area under the fluorescence peaks of various elements. Since this standard is uniform over a large sample area, this method is adequate for comparison of the beam intensity at different energies.

\section{Results}

Fig. 2 is a plot of a set of fluorescence spectra acquired using the NBS standard SRM 1833 taken with several different excitation energies. The NBS standard SRM 1833 has known amounts of the elements (K(16.2), $\mathrm{Ti}(12.2), \mathrm{Fe}(13.8), \mathrm{Zn}(3.86), \mathrm{Pb}(16.3)$, quantities in $\mu \mathrm{g} / \mathrm{cm}^{2}$ ) deposited on a $1.58 \mathrm{mg} / \mathrm{cm}^{2}$ substrate. The area under a particular fluorescence peak is proportional to the incident radiation intensity from the sample. When the energy was tuned from $6 \mathrm{keV}$ to $14 \mathrm{keV}$, the intensity varies by severalfold. Nevertheless, even at the highest energy in this experiment, the detection of all the elements that can be excited is rather straightforward. This indicates that minimum detectable limits similar to those in Ref. 10 can be achieved for this extended energy range and that the intensity is greater than $10^{\circ}$ photons $/ \mathrm{s}$ for a ring current of $100 \mathrm{~mA}$. On the low energy side, the intensity is attenuated by the beamline components (Be windows, air, filter, etc). A major improvement is possible when these absorptions are reduced (for example, by putting the setup in a He aimosphere). We will discuss the possibility of improvements in the high energy side in next section.

To demonstrate the advantage of using variable excitation energy, we have shown in Fig. 3 two spectra that were taken using $10 \mathrm{keV}$ and $6.3 \mathrm{keV} \mathrm{X}$-rays as the excitation source on a geological sample. The sample, mineral enstatite $(34 \% \mathrm{MgO}, 56 \% \mathrm{SiO}, 8.54 \% \mathrm{FeO}$, and other minerals), is believed to be a constituent of the Earth's mantle. This sample is representative of the chemical composition of the dominant minerals in the Earth. The possible chemical reactions, as well as physical transformations under the mantle conditions of high pressure and high temperature are important to our understanding of the planet. Since the high pressure and temperature can now be mimicked in the laboratory, the examination of 
possible segregation due to chemical reaction or phase transformation may provide insights into the physical processes that occur under these conditions. The determination of minor components is difficult wsing other means of microprobing because of the presence of a large amount of $\mathrm{Fe}$. It is evident that by going to a excitation energy that is lower than the Fe absorption edge, we enhanced the signal and also reduced the background. The gain in signal is about three times with a simultaneous $50 \%$ backgrourd reduction. This indicates that at least a four fold improvement in the MDL is achievable for this kind of sample. By going below the Fe edge, we also eliminated the escape peaks due to the Fe $\mathrm{K}$ fluorescence lines. The reduction in detector dead time is also of importance, because, in this kind of sample, we can easily saturate the detector system with the Fe K X-rays.

\section{Discussion}

In this section, we will look closely at the possibility of optimizing our focusing system and project the future capabilities of the X-ray microprobe. The beam size is of primary importance in our applications. Therefore we will start with a brief discussion of the beam size, then examine the intensity attainable at various excitation energies.

In the simple Kirlpatrick-Baez geometry that we adopted, when the glancing angle is small, we can consider the horizontal and vertical directional focusing separately. In each direction, the focused beam size $s$ is given by

$$
s=\frac{1}{M} S_{\text {marex }} T_{x} T_{r}
$$

where $\frac{1}{M}$ is the demagnification factor, $S_{\text {source }}$ is the apparent source size, which can be either the natural size or the size of an aperture, $T_{x}$ is the point spread function of the designed optical system, and $T_{r}$ is the effect due to random errors in the optical elements(e.g. due to surface roughness and deviation from the designed figure).

At some synchrotron radiation facilities where the source size is small, one can use the natural beam without an aperture because a large demagnification is possible due to strong collimation of the beam. For our sjstem operating at $10 \mathrm{keV}$ at beamline X26 at the National 
Syachrotron Light Source (NSLS), the demagnification is $M \approx 400$ for one of the mirrors and 300 for the other. Assuming a natural size (FWHM) of $1 \mathrm{~mm}$ by $0.4 \mathrm{~mm}$, the source size limited focused beam size would be $2.5 \mu \mathrm{m}$ by $1.3 \mu \mathrm{m}$. For the same set of multilayer mirrors, operating at different energies requires changing the incidence angles and is accompanied by changes in the mirror demagnification that affect the possible focused beam sizes. In fact, $M(E)=M\left(E_{0}\right) E / E_{0}$, so for energy lower than the designed operating energy, a smaller demagnification and hence a larger spot size results. Even so, as we shall see, this is not the limiting factor in determining the achievable sizes in the current experiments.

The requirement on $T_{r}$ is not too critical because of the small size $(2 \mathrm{~cm}$ diameter) and figure (spherical) of the mirrors used in our experiment. Actually, for even a $1 \mu \mathrm{m}$ spot size, as long as the slope error is less than $1 \mu \mathrm{m} / 5 \mathrm{~cm}=20 \mu \mathrm{rad} \approx 4$ secs of arc over the 2 cm surface, we will not be limited by this. This requirement is stringent but nevertheless achievable.

The last and most important factor is the use of spherical mirrors instead of the ideal ellipsoidal mirrors. As pointed out by Kirkpatrick and Baez [11], for a cylindrical surface ( when the glancing angle is small, one can ignore the difference between the cylindrical and spherical surfaces), the extreme rays extends over a size of $T_{x}=\frac{3}{2} W^{2} / R$, where $W$ is the half width of the reflecting surface, and $R$ is the radius of curvature. In white radiation experiments, the width $W$ does not depend on the multilayer angular bandpass, but is restricted only by physical size or apertures. For $W=1 \mathrm{~cm}, R=6 \mathrm{~m}$, we have $T_{x}=25$ $\mu \mathrm{m}$. If we take into account the intensity distribution, the region that contains $50 \%$ of total intensity would have a size of only $25 \%$ of $T_{x}$, which is about $6 \mu \mathrm{m}$. This agrees reasonably well with our previous measurement[6]. We notice that $T_{x}$ does not depend on the energy of the incident radiation; thus, a similar size for the focused beam is expected for all energies. and this represents the current spatial resolution limit. It is also clear that $T_{x}$ can be reduced by using a smaller mirror to get smaller beam sizes at the sacrifice of intensity. For focusing of monochromatic radiation, the effective mirror width is reduced by the angular bandpass of the multilayer coatings so it is possible to obtain a smaller value for $T_{x}$.

We now look at various factors that affect the focused beam intensity. The total intensity 
can be written as

$$
I=\eta \Omega B_{\text {source }} S_{\text {eff }}
$$

where $\eta$ is the efficiency, $\Omega$ is the collecting solid angle of the optical system, $B_{\text {source }}$ is the source brightness, and $S_{e f f}$ is the effective size of the source.

One of the most important factors that determine the obtainable intensity at the focusing plane is given by the source characteristics. The source size affects the focused beam size and the total intensity, but if the source size is small enough so that the focused beam size is limited by aberrations of the optical system, the product of $B_{\text {source }}$ and $S_{\text {eff }}$ as measured by photons/sec/(unit solid angle) is the figure of merit. We therefore have plotted in Fig. 4 this quantity as a function of energy for two synchrotron radiation sources. The bending magnet source represents the typical present operating conditions at NSLS and the wiggler source is for a new 6-pole 5-Tesla superconducting viggler source that is currently being installed at NSLS. It is clear that for high energy applications( $E>10 \mathrm{keV}$ ), we should be able to gain more than one order of magnitude in the achievable intensity with this new superconducting wiggler source, when it becomes avilable.

The collected solid angle $\Omega$ and efficiency $\eta$ are closely related to each other and a clear separation is impossible. For convenience, we can write

$$
\Omega=\left(\frac{2 W_{1} \sin \theta_{1}}{S_{1}}\right)\left(\frac{2 W_{2} \sin \theta_{2}}{S_{2}}\right)
$$

where $W_{1}, W_{2}$ are the half width of the illuminated area on the mirrors, $\theta_{1}, \theta_{2}$ are the glancing angles and $S_{1}, S_{2}$ are the distances between the source and mirrors. This way we attribute all the effects due to multilayer coating to the efficiency $\eta$. We can increase $\Omega$ and thereby $I$ by increasing $W_{1}, W_{2}, \theta_{1}$ and $\theta_{2}$. However, the value of $\Omega$ is strongly influenced by the multilayer coatings. An increase in $\Omega$ often results in the decrease of $\eta$. The efficiency $\eta$ is essentially the averaged reflectivity on the illuminated surface. Due to the curvature of the mirror, the variation in the glancing angles on different parts of the mirrors is quite large compared to the angular bandpass of a uniform multilayer at a given energy, and the average reflectivity depends strongly on the shape and size of the mirrors as well as the glancing angles. For the case where the curvature of the mirrors gives a larger variation 
than the multilayer bandpass and a uniform multilayer coating is used, a weighted integral reflectivity for a given energy is proportional to the average reflectivity at this energy. For focusing monochromatic radiation, one can improve the average reflectivity by depositing laterally graded multilayer coatings (with different $d$ spacings across the mirror surface) so the maximum reflectivity can be achieved at all points. For focusing white radiation when the energy resolution is not important (as in most $\mathrm{X}$-ray fluorescence microprobe experiments), this lateral grading is not necessary because the maximum reflectivity can be achieved at each point by $\mathrm{X}$-rays of a slightly different energy. In this case, the integral reflectivity for all energies at a given angle is important. This can be usually improved by using depth-graded multilayers (i.e. the $d$ spacing varies for different layer pairs).

In all these cases, a knowledge of the multilayer reflectivity as a function of both energy and angle is important. For illustration purposes, we plotted in Fig. 5 the calculated reflectivity of a $25 \mathrm{~A} \mathrm{~W} / \mathrm{C}(35 \% \mathrm{~N}, 65 \% \mathrm{C})$ uniform planar multilayer coating for different energies near the first Bragg angle. In this calculation, a sharp interface was assumed. If a more diffuse interface is present, the Bragg peak reflectivity will degrade somewhat, but the relative change in the reflected intensity will be changed to a much smaller extent. One can see, except for the brief period near the $W \mathrm{~L}$ edges, the W/C multilayer consistently provides high reflectivity in the 6 to $20 \mathrm{keV}$ range. This indicates that a microprobe with excitation radiation of energy in the vicinity of $20 \mathrm{keV}$ is possible. The optimization of a design for a particular application depends on how closely one can predict multilayer's reflectivity, especially for small $d$ spacing multilayers where the interface effects are quite large

\section{Conclusion}

We have shown that a simple Kirkpatrick-Baez type focusing device, when used on a polychromatic (white) synchrotron radiation beamline, can be iuned in the energy range from $6 \mathrm{keV}$ to $14 \mathrm{keV}$ at least. The spatial resolution of this microprobe at different energies is $\langle 10 \mu \mathrm{m}$. The intensity varies within a factor of 10 or so when one goes from the low 
energy end to high energy end with the NSLS bending magnet source. The tunability is very useful when one wants to avoid excitation of particular elements.

For radiations of energy higher than $14 \mathrm{keV}$, the superconducting wiggler source at NSLS will provide more than one order of magnitude enhancement in intensity compared with the existing bending magnet sources. This should provide a good opportunity for both high energy $\mathrm{X}$-ray fluorescence microprobe and diffraction microprobe experiments in the future.

Good reflectivity is expected from ideal multilayer coatings at quite high energy ( $>20$ $\mathrm{keV}$ ). A good model that takes into account the graded interfaces in the multilayer coatings would be very usefu' in the optimization of a X-ray microprobe using multilayer coated mirrors.

\section{Acknowledgement:}

We would like to thank P. Batson and R. Delaro for their mechanical work on the X-ray microprobe The authors are grateful to X.Y. Li for providing the enstatite samples used in this experiment and J. Davis for providing the X-ray optical constants for $W$ and $C$. This work was supported by the Director, Office of Energy Research, Office of Basic Energy Science, Materials Sciences Division, of the U.S. Department of Energy under Contract No. AC03-76SF00098. 


\section{Figure Captions:}

Fig. 1. Schematic of the X-ray fluorescence microprobe setup. Central to the system are the two multilayer coated mirrors. The system also includes an optical microscope to assist in the positioning of samples.

Fig. 2. The fluorescence spectra from NBS standard SRM 1833 excited by X-rays of different energies.

Fig. 3. The $\mathrm{X}$-ray fluorescence spectra from enstatite as excited by $\mathrm{X}$-rays of energies 6.3 $\mathrm{keV}$ and $10 \mathrm{keV}$. As the energy is reduced to $6.3 \mathrm{keV}$, the Fe $\mathrm{K}$ escape peaks disappear ard the background is reduced.

Fig. 4. A comparison of calculated brightness for a bending magnet radiation source and the superconducting wiggler source at NSLS in the energy range $5 \sim 25 \mathrm{keV}$. The increase in brightness is at least a factor of 10 for energies greater than $15 \mathrm{keV}$.

Fig. 5. The calculated reflectivities at different energies for a planar W/C multilayer. High reflectivities are expected for the whole 6 to $20 \mathrm{keV}$ range except for energies very close to the tungsten $L$ edges where severe absorption results in a relatively low reflectivity. 


\section{References:}

[1] C. J. Spariss Jr., in: "Synchrotron Radiation Research", H. Winick and S. Doniach, ed., Plenum Press, New York(1980).

[2] A. Iida, K. Sakurai, T. Matsushita and Y. Gohshi, Nucl. Instr. ond Meth. 228(1985)556.

[3] W. F terson, P. Ketelsen, A. Knochel and R. Pausch, Nucl. Instr. and Meth. A246(1986)731.

[4] K.W. Jones and Barry M. Gordon, Anal. Chem. 61(1989)341A.

[5] A.C.Thompson, Y. Wu, J.H. Underwood and T.W. Barbee Jr., Nucl. Instr. an. $\dot{\alpha}$ Meth. A225(1987)603.

[6] J.H. Underwood, A.C. Thompson. Y. WL and R.D. Giauque, Nucl. Instr. cad Meth. A226(1988)296.

[7] G.E. Ice, Nucl. Instr. and Meth. B24/25(1987)397.

[8] J.A. Xu, H.K. Mao and P.M. Bell, Science 232(1986)1404; William C. Moss, John O. Hallquist, Robin Reichlis, Fenneth A. Goettel and Sue Martin, Appl. Phys. Lett. 48(1986)1258; H. K. Mao, Y. Wu, R. J. Hemley, L. C. Chen, J. F. Shu, and L. W. Finger, Science 246(1989)649.

[9] A.C. Thompson, J.H. Underwood, Y. Wu, R.D. Giauque, K.W. Jones and M.L. Rivers, Nucl. Instr. and Meth. A226(1988)318.

[I0] R.D. Giauque, A.C. Thompson, J.H. Underwood, Y. Wu, K.W. Jones and M.L. Rivers, Anal. Chem. 60(1988)885.

[11] P. Kirl.patrick and A. V. Baez, J. Opt. Soc. Am. 39(1948)766. 


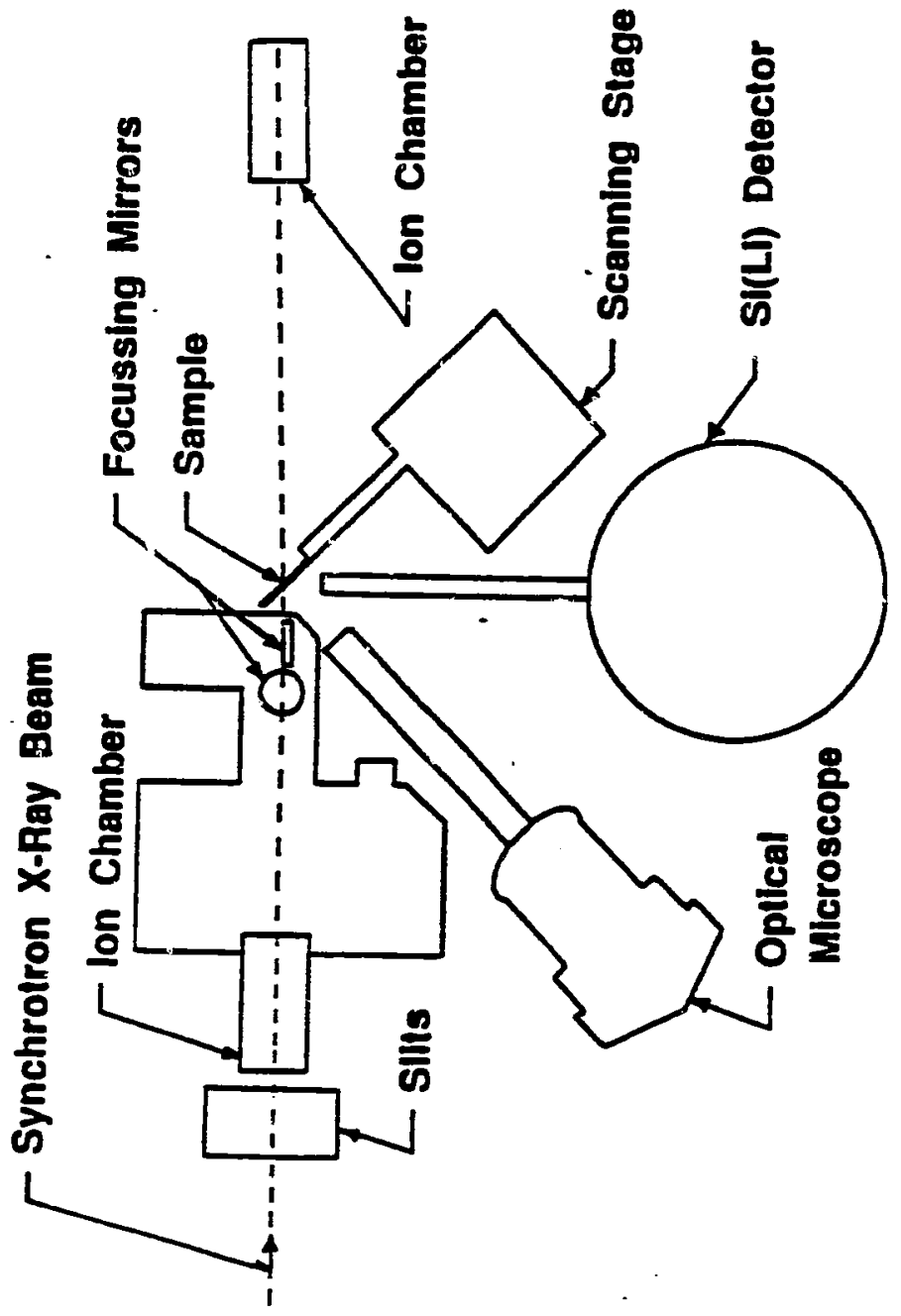




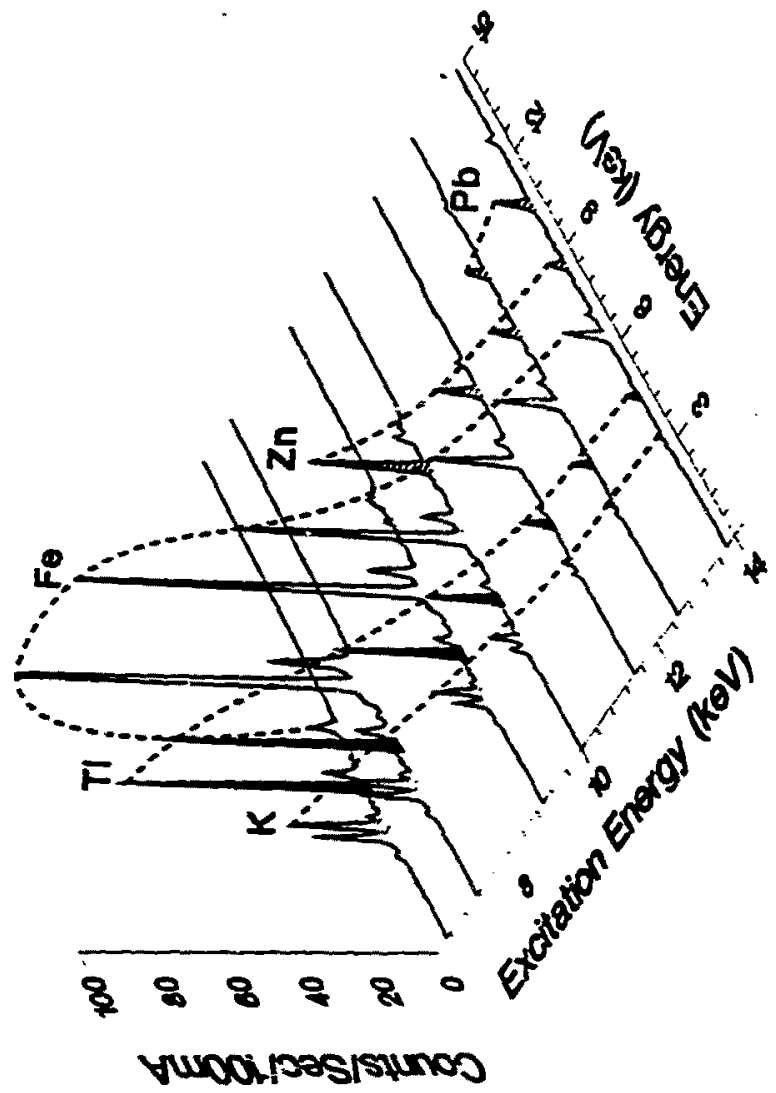




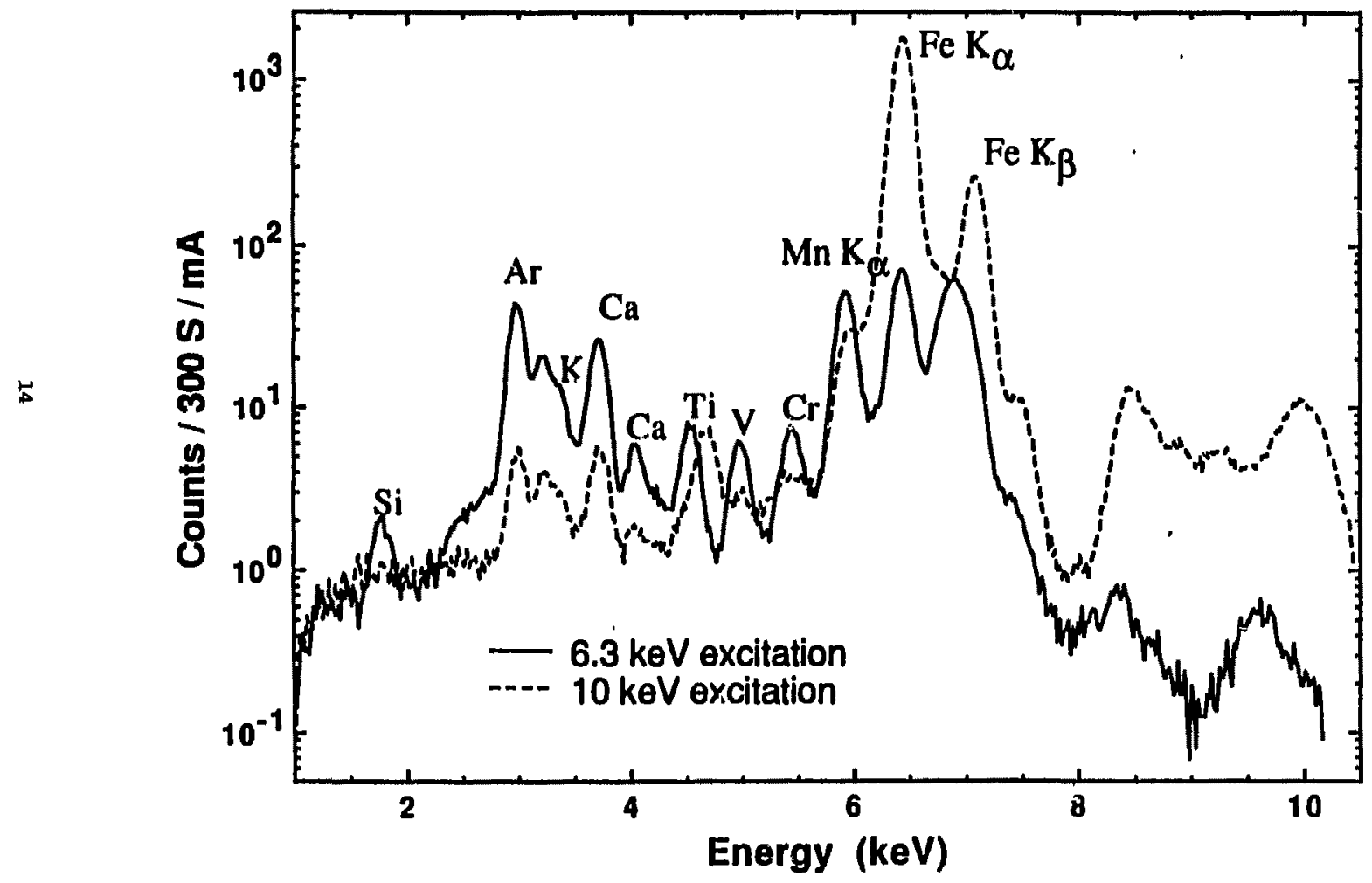




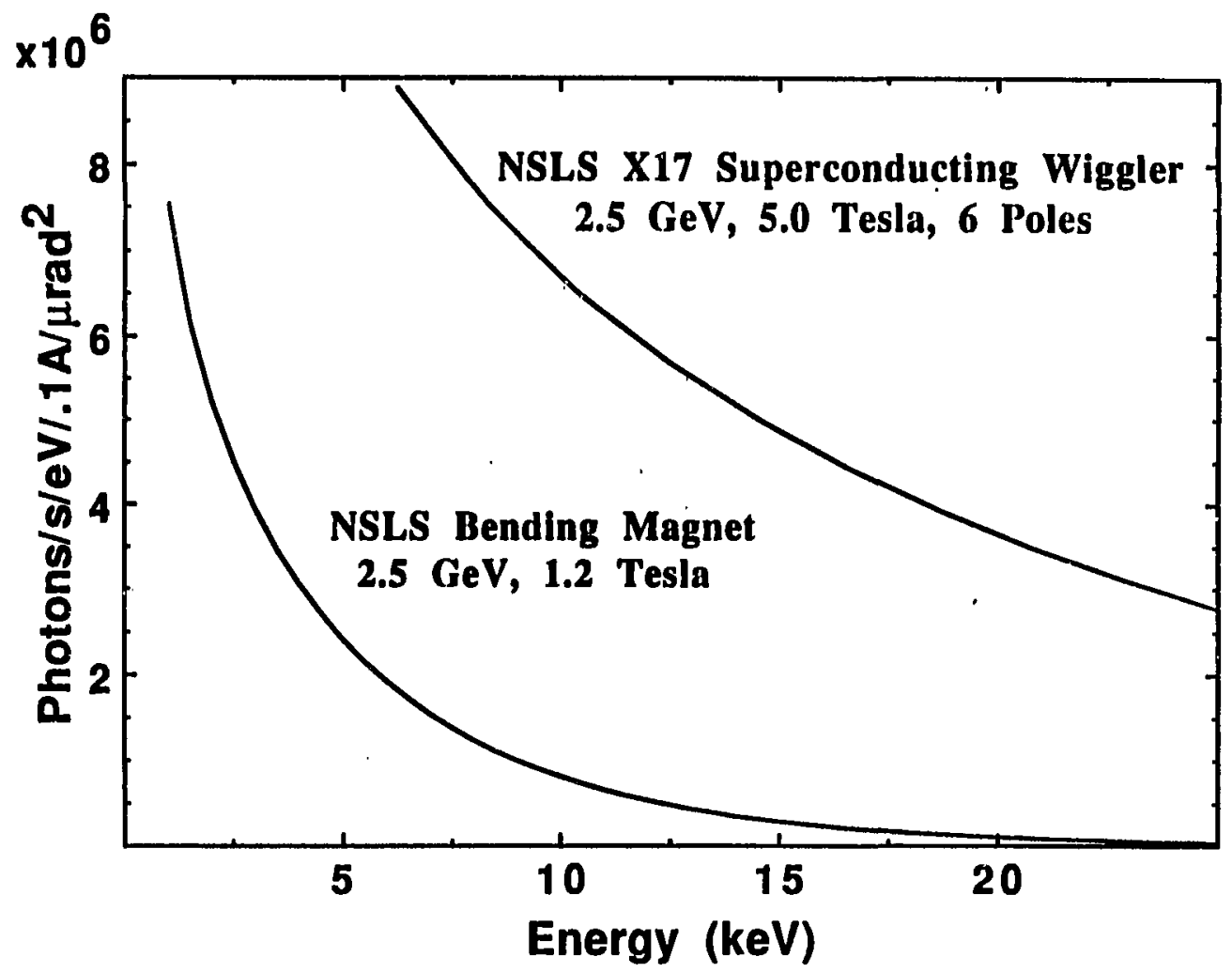




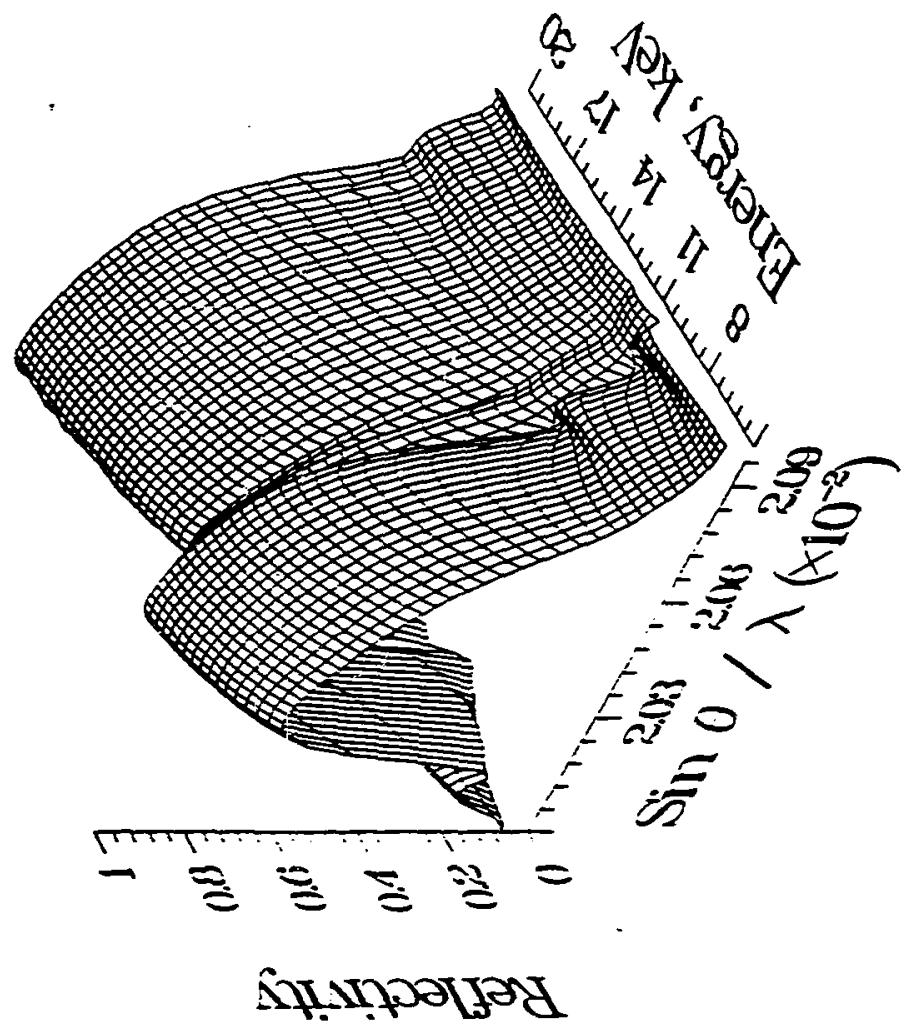

